A. Fujii

Nagoya Math. J.

Vol. 64 (1976), 149-158

\title{
ON SOME ANALOGUES OF TITCHMARSH DIVISOR PROBLEM
}

\author{
AKIO FUJII
}

\section{§1. Introduction}

In [15] Titchmarsh posed and solved under the generalized Riemann Hypothesis, the problem of an asymptotic behavior of the number of the solutions of the equation $1=p-n_{1} n_{2}$ for a prime $p \leq x$ and natural numbers $n_{1}$ and $n_{2}$. When we put $\tau(n)=\sum_{a \mid n} 1$, then the above problem is to get an asymptotic law for the sum

$$
\sum_{p \leq x} \tau(p-1) \text {. }
$$

Later Linnik [11] solved this unconditionally using his dispersion method. The proof without the dispersion method is also known (Cf. [3] and [14]). Here we are concerned with an asymptotic behavior of the sum

$$
\sum_{p_{1} \leq x^{\delta}, p_{2} \leq x^{1-\delta}} \tau\left(p_{1} p_{2}-1\right),
$$

where $p_{1}$ and $p_{2}$ run over primes and $\delta$ is in $0<\delta \leq 1 / 2$. Linnik's dispersion method solves this for $0<\delta<1 / 6$. But it does not work for other values of $\delta$. Barban [1] solved this for $\delta=1 / 2$. Here we shall prove

Theorem 1. Suppose that $\delta$ is in $0<\delta \leq 1 / 2$ and $\delta \log x$ tends to $\infty$ as $x$ tends to $\infty$. Then we have

$$
\begin{aligned}
\sum_{p_{1} \leq x^{\delta}, p_{2} \leq x^{1-\delta}} \tau\left(p_{1} p_{2}-1\right)= & \frac{315}{2 \pi^{4}} \frac{\zeta(3)}{\delta(1-\delta)} \frac{x}{\log x} \\
& +O\left(x \delta^{-1}(\log x)^{-2}\left(\log \log x+\delta^{-1}\right)\right)
\end{aligned}
$$

uniformly for $\delta$, where $\zeta(s)$ is the Riemann zeta function.

We shall also prove

Received March 18, 1976. 
THEOREM 2.

$$
\sum_{p_{1} p_{2} \leq x} \tau\left(p_{1} p_{2}-1\right)=315 \pi^{-4} \zeta(3) x \log \log x+O(x) .
$$

To prove our theorems we need the following mean value theorems. We shall state them in the more general form than we need in this paper. For simplicity we put

$$
E(y ; a, d)=\sum_{\substack{p \leq y \leq \\ p \equiv a(\bmod d)}} \cdot 1-\frac{\operatorname{Li}(y)}{\varphi(d)},
$$

where

$$
\operatorname{Li}(y)=\int_{2}^{y} \frac{d x}{\log x}+O(1)
$$

and $\varphi(d)$ is the Euler function. Then we shall prove

THEOREM 3. Suppose that $\sum_{m \leq x}|b(m)|^{2} \ll x(\log x)^{C}$ with some positive absolute constant $C$. Then for any positive constants $A$ and $b(<1)$, there exists a positive constant $B$ such that

$$
\sum_{d \leq Q} \operatorname{Max}_{(a, d)=1}\left|\sum_{\substack{1 \leq m \leq x \\(m, d)=1}} b(m) E\left(x^{1-\delta} ; a m^{*}, d\right)\right| \ll x(\log x)^{-A}
$$

uniformly for $\delta$ in $0 \leq \delta<1-(\log x)^{-b}$, where $Q=x^{1 / 2}(\log x)^{-B}$ and $m m^{*} \equiv 1(\bmod d)$.

The conclusion still holds even if we replace $E\left(x^{1-\delta} ; a m^{*}, d\right)$ by $E\left(x / m ; a m^{*}, d\right)$. We call this Theorem $3^{\prime}$. In $\S 2$ we shall list up and prove some lemmas. We shall prove Theorem 3 in $\S 3$, Theorem 1 in $\S 4$ and Theorem 2 in $\S 5$. We shall also give some remarks in $\S 6$.

\section{§2. Some lemmas}

LEMma 1. For an arbitrarily given small positive $\varepsilon$, for all $d \ll x^{1-s}$ and $(a, d)=1$, we have

$$
\sum_{\substack{p q \leq x \\ p q \equiv a(\bmod d)}} \cdot 1 \ll \frac{x \log \log x}{\varphi(d) \log x},
$$

where $p$ and $q$ run over primes.

Proof. Let $\eta$ be a small positive number less than $\varepsilon / 2$. Now the left hand side is 


$$
\ll \sum_{\substack{x \eta<p, q \\ p q \leq x \\ p q \equiv a(\bmod d)}} 1+\sum_{\substack{p \leq x \\(p, d)=1}} \sum_{\substack{q \leq x / p \\\left(p \equiv a p^{*}(\bmod d)\right.}} 1=\sum_{1}+\sum_{2},
$$

say.

$$
\sum_{1} \ll x /(\varphi(d) \log x)
$$

by Selberg's sieve method as usual.

$$
\sum_{2} \ll \sum_{p \leq x^{\eta}} x /(p \log x \cdot \varphi(d)) \ll x \log \log x /(\varphi(d) \log x)
$$

by the Brun-Titchmarsh theorem. Hence we get our conclusion. Q.E.D.

LEMMA 2. Let $m$ be an integer different from 1 . Then we have

$$
\left|\sum_{\chi: d}^{*} \chi(m)\right| \leq|(m-1, d)|,
$$

where in the summation, $\chi$ runs over all primitive characters mod $d$.

Proof. We denote the sum in the left hand side by $S^{*}(d, m)$. We put $S(d, m)=\sum_{\chi \neq \chi_{0}} \chi(m)$, where $\chi$ runs over all non-principal characters $\bmod d$. We know for $(m, d)=1$,

$$
S(d, m)= \begin{cases}\varphi(d)-1 & \text { if } m \equiv 1(\bmod d) \\ -1 & \text { otherwise } .\end{cases}
$$

Suppose that $d=\prod p^{\nu}$ and $(m, d)=1$. Then $S^{*}(d, m)=\prod_{p \mid d} S^{*}\left(p^{\nu}, m\right)$. Suppose that $p \neq 2$. We denote the primitive character attached to $\chi$ by $\chi^{*}$. Then

$$
\begin{aligned}
S\left(p^{\nu}, m\right) & =\sum_{\chi \neq x_{0}} \chi(m)=\sum_{\chi \neq x_{0}} \chi^{*}(m)=\sum_{\nu \geq j \geq 1} S^{*}\left(p^{j}, m\right) \\
& =S^{*}\left(p^{\nu}, m\right)+S\left(p^{\nu-1}, m\right) .
\end{aligned}
$$

Hence for $\nu \geq 1$,

$$
\begin{aligned}
S^{*}\left(p^{\nu}, m\right) & =S\left(p^{\nu}, m\right)-S\left(p^{\nu-1}, m\right) \\
& = \begin{cases}\varphi\left(p^{\nu}\right)-\varphi\left(p^{\nu-1}\right) & \text { if } p^{\nu} \mid m-1 \\
-\varphi\left(p^{\nu-1}\right) & \text { if } p^{\nu-1} \| m-1 \\
0 & \text { otherwise }\end{cases}
\end{aligned}
$$

Next for $p=2$ and for $\nu \geq 2$,

$$
S\left(2^{\nu}, m\right)=\sum_{\nu \geq j \geq 2} S^{*}\left(2^{j}, m\right)=S^{*}\left(2^{\nu}, m\right)+S\left(2^{\nu-1}, m\right) .
$$


Hence for $\nu \geq 1$, we get

$$
S^{*}\left(2^{\nu}, m\right)= \begin{cases}\varphi\left(2^{\nu}\right)-\varphi\left(2^{\nu-1}\right) & \text { if } 2^{\nu} \mid m-1 \\ -\varphi\left(2^{\nu-1}\right) & \text { if } 2^{\nu-1} \| m-1 \\ 0 & \text { otherwise }\end{cases}
$$

Hence we have

$$
\begin{aligned}
\left|S^{*}(d, m)\right| & \leq \prod_{p \mid d}\left|S^{*}\left(p^{\nu}, m\right)\right| \\
& \leq \prod_{p^{\nu \mid m-1}}\left(\varphi\left(p^{\nu}\right)-\varphi\left(p^{\nu-1}\right)\right) \prod_{p^{\nu-1} \| m-1} \varphi\left(p^{\nu-1}\right) \\
& \leq \prod_{p^{\nu \mid m-1}} p^{\nu} \prod_{p^{\nu-1 \| m-1}} p^{\nu-1} \\
& \leq|(m-1, d)| .
\end{aligned}
$$

LEMMA 3.

$$
\sum_{D<d \leq Q} \frac{1}{\varphi(d)} \sum_{\chi: d}^{*}\left|\sum_{n=M+1}^{M+N} a_{n} \chi(n)\right|^{2} \ll\left(Q+\frac{N}{D}\right) \sum_{n=M+1}^{M+N}\left|a_{n}\right|^{2}
$$

(Cf. (10) of [6] or (3) of [2]).

LEMmA 4. For $d \leq x^{1 / 2+s}$, and for $(a, d)=1$,

$$
\sum_{\substack{n \leq x \\ n \equiv a(\bmod d)}} \tau^{2}(n) \ll x\left(\prod_{p \mid d}\left(1-p^{-1}\right) \cdot \log (x / d)\right)^{3} / d .
$$

(Cf. Lemma 1.1 .3 of [11]).

LEMMA 5. For all $d \ll x^{2 / 3-s},(a, d)=1$ and for $0<\delta \leq 1 / 2$,

$$
\sum_{\substack{p q=a(\bmod d)>\\ p \leq x^{\delta}, q \leq x^{1}-\delta}} \cdot 1 \ll \delta^{-1} x /\left(\varphi(d)(\log x)^{2}\right) .
$$

(Cf. Lemma 3.6 of [1])

\section{§3. Proof of Theorem 3 and $3^{\prime}$}

3-1. We shall prove only Theorem 3 since Theorem $3^{\prime}$ can be proved in a similar manner (Cf. [4] and [5]). By Bombieri's mean value theorem we may suppose that $\delta \geq A^{\prime} \log \log x / \log x$ for a sufficiently large constant $A^{\prime}$. For simplicity we put $x^{\prime}=x^{\delta}, x^{\prime \prime}=x^{1-\delta}, \ell=\log x$ and $\pi(x, \chi)$ $=\sum_{p \leq x} \chi(p)$. We also put $Q=x^{1 / 2} \ell^{-B}$ with sufficiently large $B$ which will be chosen appropriately in the following, $Q_{j}=2^{j} \ell^{D}$ for $j=0,1,2$, $\cdots, J$, where $J$ satisfies $2^{J-1} \ell^{D}<Q \leq 2^{J} \ell^{D}$ and $D$ is a sufficiently large 
constant. We always denote an arbitrarily small positive number by $\varepsilon$, a sufficiently large constant by $E$ and some positive absolute constants by $C$. Now for $d \leq Q,(m, d)=1$ and $(a, d)=1$,

$$
\begin{aligned}
E\left(x^{\prime \prime} ; a m^{*}, d\right)= & \frac{1}{\varphi(d)} \sum_{\chi \neq \chi_{0}} \bar{\chi}(a) \chi(m) \pi\left(x^{\prime \prime}, \chi\right) \\
& +\frac{1}{\varphi(d)}\left(\sum_{p \leq x^{\prime \prime}} \cdot 1-\mathrm{Li} x^{\prime \prime}\right)-\frac{1}{\varphi(d)} \sum_{\substack{p \leq x^{\prime \prime} \\
p \mid d}} \cdot 1 \\
= & \frac{1}{\varphi(d)} \sum_{\chi \neq \chi_{0}} \bar{\chi}(a) \chi(m) \pi\left(x^{\prime \prime}, \chi\right)+O\left(x^{\prime \prime} \ell^{-E} \varphi(d)^{-1}\right)
\end{aligned}
$$

by the prime number theorem. Since

$$
\pi\left(x^{\prime \prime}, \chi^{*}\right)-\sum_{\substack{p \mid d \\ p \nmid d^{*} \\ p \leq x^{\prime \prime}}} \chi^{*}(p)=\pi\left(x^{\prime \prime}, \chi\right)
$$

we have

$$
\begin{aligned}
& \sum_{d \leq Q} \operatorname{Max}_{(a, d)=1}\left|\sum_{\substack{m \leq x^{\prime} \\
(m, d)=1}} b(m) E\left(x^{\prime \prime} ; a m^{*}, d\right)\right| \\
& \ll \sum_{d \leq Q} \frac{1}{\varphi(d)} \sum_{\chi \neq \chi_{0}}\left|\sum_{\substack{m \leq x^{\prime} \\
(m, d)=1}} b(m) \chi^{*}(m) \pi\left(x^{\prime \prime}, \chi^{*}\right)\right| \\
& +\sum_{d \leq Q} \frac{1}{\varphi(d)} \operatorname{Max}_{(a, d)=1}\left|\sum_{x \neq \chi_{0}} \bar{\chi}^{*}(a) \sum_{\substack{m \leq x^{\prime} \\
(m, d)=1}} b(m) \chi^{*}(m) \sum_{\substack{p \mid d \\
p \nmid d^{*} \\
p \leq x^{\prime \prime}}} \chi^{*}(p)\right| \\
& +x \ell^{-E}=S_{1}+S_{2}+x \ell^{-E} \text {, }
\end{aligned}
$$

say, where $d^{*}$ is the conductor of $\chi$.

3-2. We shall estimate $S_{2}$ first. Using Lemma 2, we get

$$
\begin{aligned}
S_{2} & \ll \sum_{d \leq Q} \frac{1}{\varphi(d)} \operatorname{Max}_{(a, d)=1} \sum_{\substack{d^{*} \mid d \\
d^{*}>1}} \sum_{\substack{p|d| d d^{*} \\
p \leq x^{\prime \prime}}} \sum_{\substack{m \leq x^{\prime} \\
(m, d)=1}}|b(m)|\left|\sum_{\substack{x: d^{*} \\
*}}^{*} \chi\left(a^{*} m p\right)\right| \\
& \ll \sum_{d \leq Q} \frac{1}{\varphi(d)} \operatorname{Max}_{(a, d)=1} \sum_{\substack{d^{*} \mid d \\
d^{*}>1}} \sum_{\substack{p \mid d / d d^{*} \\
p \leq x^{\prime \prime}}} \sum_{\substack{m \leq x^{\prime} \\
(m, d)=1}}|b(m)|\left|\left(m p-a, d^{*}\right)\right| \\
& \ll \ell^{C} \operatorname{Max}_{1 \leq a \leq Q} S_{a},
\end{aligned}
$$

where we put

$$
S_{a}=\sum_{k_{1} k_{2} \leq Q} 1 /\left(k_{1} k_{2}\right) \sum_{\substack{p \mid k_{2} \\ p \leq x^{\prime \prime} \\(a, p)=1}} \sum_{m \leq x^{\prime}}|b(m)|\left|\left(m p-a, k_{1}\right)\right| .
$$




$$
\begin{aligned}
S_{a} & \ll \sum_{k_{1} \leq Q} 1 / k_{1} \sum_{\substack{m \leq x^{\prime}, p \leq x^{\prime \prime} \\
(a, p)=1}}|b(m)|\left|\left(m p-a, k_{1}\right)\right| \sum_{\substack{k_{2} \leq Q \\
p \mid k_{2}}} 1 / k_{2} \\
& \ll \ell^{C} \sum_{\substack{m \leq x^{\prime}, p \leq x^{\prime \prime} \\
(a, p)=1}}|b(m)| / p \sum_{d|| m p-a \mid} d \sum_{d \mid k_{1}, k_{1} \leq Q} 1 / k_{1} \\
& \ll \ell^{C} \sum_{\substack{m \leq x^{\prime}, p \leq x^{\prime \prime} \\
(a, p)=1}}|b(m)| \tau(|m p-a|) / p \\
& \ll \ell^{C} x^{\prime 1 / 2}\left(\sum_{m \leq x^{\prime}} \sum_{\substack{p \leq x^{\prime \prime} \\
(a, p)=1}} \tau^{2}(|m p-a|) / p\right)^{1 / 2} \\
& \ll \ell^{C} x^{\prime 1 / 2} S_{a}^{\prime 1 / 2},
\end{aligned}
$$

say. If $0<\delta \leq 1 / 2$, then $S_{a}^{\prime} \ll x^{s} \ell^{c} x^{\prime} \ll x$.

If $1 / 2 \leq \delta<1$, then

$$
S_{a}^{\prime} \ll \sum_{\substack{p \leq x^{\prime \prime} \\(a, p)=1}} 1 / p \sum_{m \leq x^{\prime}} \tau^{2}(|m p-a|) \ll x \ell^{C}
$$

using Lemma 4. Hence always we get $S_{a} \ll x^{(1+\delta) / 2} \ell^{C}$, and

$$
S_{2} \ll x^{(1+\delta) / 2} \ell^{C} \ll x \ell^{-E} \quad \text { uniformly for } 0<\delta \leq 1-(\log x)^{-b} \text {. }
$$

3-3. Next we shall estimate $S_{1}$.

$$
S_{1} \ll \ell \operatorname{Max}_{1<b \leq Q} S_{1, b},
$$

where we put

$$
S_{1, b}=\sum_{0 \leq j \leq J} S_{1, b}(j)
$$

with

$$
S_{1, b}(j)=\sum_{Q_{j-1}<d \leq Q_{j}} \frac{1}{\varphi(d)} \sum_{\chi: d}^{*}\left|\sum_{\substack{m \leq x^{\prime} \\(m, b)=1}} \chi(m) b(m) \pi\left(x^{\prime \prime}, \chi\right)\right|
$$

for $0 \leq j \leq J$ and $Q_{-1}=1$.

By Siegel-Walfisz theorem (Cf. p. 134 and 144 of [13]), we get

$$
S_{1, b}(0) \ll x \ell^{-E} .
$$

Now

$$
\begin{aligned}
S_{1, b}(j) \ll & \left(\sum_{Q_{j}-1<d \leq Q_{j}} \frac{1}{\varphi(d)} \sum_{\chi: d} *\left|\sum_{m \leq x^{\prime}} \chi(m) b(m)\right|^{2}\right)^{1 / 2} \\
& \cdot\left(\sum_{Q_{j-1}<d \leq Q_{j}} \frac{1}{\varphi(d)} \sum_{\chi: d}^{*}\left|\pi\left(x^{\prime \prime}, \chi\right)\right|^{2}\right)^{1 / 2} \\
= & \sum_{1}^{1 / 2} \sum_{2}^{1 / 2},
\end{aligned}
$$


say. By Lemma 3, we get

$$
\sum_{1} \ll\left(Q_{j}+x^{\prime} Q_{j}^{-1}\right) \sum_{m \leq x^{\prime}}|b(m)|^{2} \ll x^{\prime} \ell^{C}\left(Q_{j}+x^{\prime} Q_{j}^{-1}\right) .
$$

Similarly we get

$$
\sum_{2} \ll\left(Q_{j}+x^{\prime \prime} Q_{j}^{-1}\right) x^{\prime \prime} .
$$

Hence $S_{1, b}(j) \ll x \ell^{-E}$ by taking $B$ and $D$ sufficiently large. Hence $S_{1} \ll x \ell^{-E}$. Combining this with the estimate of $S_{2}$, we get our conclusion.

Q.E.D.

\section{§4. Proof of Theorem 1}

We put for simplicity $x_{1}=x^{\delta}, x_{2}=x^{1-\delta}, \quad \ell=\log x$ and $Q=x^{1 / 2} \ell^{-B}$ with a sufficiently large constant $B$. Now

$$
\begin{aligned}
\sum_{p_{i} \leq x_{i}} \tau\left(p_{1} p_{2}-1\right) & =\sum_{p_{i} \leq x_{i}} \sum_{d \mid p_{1} p_{2}-1} \cdot 1 \\
& =2 \sum_{d \leq Q} \sum_{\substack{p_{i} \leq x_{i} \\
d \mid p_{1} p_{2}-1}} \cdot 1+O\left(\sum_{\substack{Q<d \leq \sqrt{x} \\
d \mid p_{1} p_{2}-1 \\
p_{i} \leq x_{i}}} \cdot 1\right) \\
& =\sum_{1}+O\left(\sum_{2}\right),
\end{aligned}
$$

say.

$$
\begin{aligned}
\sum_{1}= & 2 \sum_{d \leq Q} \sum_{\substack{p_{1} \leq x_{1} \\
\left(p_{1}, d\right)=1}}\left(\sum_{\substack{p_{2} \leq x_{2} \\
p_{2} \equiv p_{1}^{*}(\bmod d)}} \cdot 1-\operatorname{Li} x_{2} / \varphi(d)\right) \\
& +2 \sum_{d \leq Q} \sum_{\substack{p_{1} \leq x_{1} \\
\left(p_{1}, d\right)=1}} \operatorname{Li} x_{2} / \varphi(d) \\
= & O\left(x \ell^{-A}\right)+2 \operatorname{Li} x_{1} \operatorname{Li} x_{2}\left(\sum_{d \leq Q} \frac{1}{\varphi(d)}\right) \\
= & \operatorname{Li} x_{1} \operatorname{Li} x_{2} 315 \zeta(3)\left(2 \pi^{4}\right)^{-1} \log x \\
& +O\left(\operatorname{Li} x_{1} \operatorname{Li} x_{2} \log \log x\right) .
\end{aligned}
$$

On the other hand by Lemma 5 ,

$$
\sum_{2} \ll \delta^{-1} x \log \log x /(\log x)^{2} \quad \text { uniformly for } 0<\delta \leq 1 / 2 .
$$

Hence we get our conclusion.

Q.E.D.

Remark. To prove our theorem 1 just for any $\delta$ in $0<\delta<1 / 2$ we do not need Barban's Lemma 3.6 (namely, Lemma 5 in §2). Because by the Brun-Titchmarsh theorem we get

$$
\sum_{2} \ll x \log \log x /(\log x)^{2} .
$$




\section{§5. Proof of Theorem 2}

Let $\delta$ be any number in $0<\delta<1$. We put $x^{\prime}=x^{\delta}, x^{\prime \prime}=x^{1-\delta}, Q=$ $x^{1 / 2}(\log x)^{-B}$ and $F(\delta, x)=\sum_{p \leq x^{\delta}}(p \log (x / p))^{-1}$. Now

$$
\begin{aligned}
\sum_{p q \leq x} \tau & (p q-1) \\
& =\sum_{\substack{p \leq x^{\prime} \\
p q \leq x}} \tau(p q-1)+\sum_{\substack{q \leq x^{\prime \prime} \\
p q \leq x}} \tau(p q-1)-\sum_{\substack{p \leq x^{\prime} \\
q \leq x^{\prime \prime}}} \tau(p q-1) \\
& =\sum_{1}+\sum_{2}-\sum_{3},
\end{aligned}
$$

say. By Theorem $1 \sum_{3} \ll x(\log x)^{-1}$.

$$
\begin{aligned}
\sum_{1}= & 2 \sum_{d<Q} \sum_{\substack{p \leq x^{\prime} \\
(p, d)=1}} \operatorname{Li}(x / p) / \varphi(d) \\
& +O\left(\sum_{d \leq Q} \sum_{\substack{p \leq x^{\prime} \\
(p, d)=1}}\left(\sum_{\substack{q \leq x / p \\
q \equiv p^{\leqslant}(\bmod d)}} \cdot 1-\operatorname{Li}(x / p) / \varphi(d)\right)\right) \\
& +O\left(\sum_{Q<d \leq \sqrt{x}} \sum_{\substack{p \leq x \leq \\
p q \equiv 1(\bmod d)}} \cdot 1\right) \\
= & 315 \zeta(3)\left(2 \pi^{4}\right)^{-1} x \log x F(\delta, x)+O(x \log \log x F(\delta, x)) \\
& +O\left(x(\log \log x)^{2}(\log x)^{-1}\right) \\
& +O\left(x(\log x)^{-1} \sum_{d<Q} \frac{1}{\varphi(d)} \cdot \sum_{\substack{p \leq x^{\prime} \\
p \nmid d}} \frac{1}{p}\right) .
\end{aligned}
$$

The last term is $\ll x$. In a similar way we get

$$
\begin{aligned}
\Sigma_{2}= & 315\left(2 \pi^{4}\right)^{-1} \zeta(3) x \log x F(1-\delta, x) \\
& +O(x \log \log x F(1-\delta, x))+O(x) .
\end{aligned}
$$

Now

$$
\begin{aligned}
F(\delta, x) & =\int_{3 / 2}^{x^{\prime}} \frac{1}{t \log (x / t)} d\left(\sum_{p \leq t} 1\right) \\
& =(\log \log x+\log \delta-\log (1-\delta)) / \log x+O\left((\log x)^{-1}\right)
\end{aligned}
$$

Hence

$$
F(\delta, x)+F(1-\delta, x)=2 \log \log x / \log x+O(1 / \log x) .
$$

Hence we get our conclusion.

Q.E.D.

\section{§6. Concluding remarks}

6-1. Theorem 1 and 2 for the sum of $\tau\left(N-p_{1} p_{2}\right)$ or $\tau\left(p_{1} p_{2}-a\right)$ can be similarly proved. 
6-2. More generally, if $k \geq 1, \delta_{1}+\delta_{2}+\cdots+\delta_{k}=1, \delta_{i}>0$ for each $i$ and $\delta_{j}+\delta_{\ell}>3 / 4$ for some $j, \ell$ in $1 \leq j, \ell \leq k$, then we have

$$
\begin{aligned}
\sum_{p_{i} \leq x^{\delta i}} \tau\left(p_{1} p_{2} \cdots p_{k}-1\right) \\
\quad=\frac{315}{2 \pi^{4}} \frac{\zeta(3)}{\delta_{1} \delta_{2} \cdots \delta_{k}} \frac{x}{(\log x)^{k-1}}+O\left(x \log \log x /(\log x)^{k}\right) .
\end{aligned}
$$

For $k=2$, this is nothing but our Theorem 1. (Cf. [1] and [9] for previous weaker results.).

Further, under the same condition of $\delta_{1}, \delta_{2}, \cdots, \delta_{k}$, we have an asymptotic formula for the sum

$$
\sum_{p_{i} \leq x^{\delta} i} \tau_{m}\left(p_{1} p_{2} \cdots p_{k}-a\right) \quad \text { for almost all } a
$$

and for each $m \geq 3$, where

$$
\tau_{m}(n)=\sum_{n=d_{1} d_{2} \cdots d_{m}} \cdot 1 .
$$

(Cf. [16] for $k=1$ and for $m \geq 3$.)

6-3. In a similar manner to the proof of Theorems 3 and $3^{\prime}$, we get the following inequality; for any positive constants $A$ and $b(<1)$, if $\sum_{m \leq x}|b(m)|^{2} \ll x(\log x)^{C}, b(m) \ll x^{1-\delta-\beta}$ for $m \leq x^{\delta}, \beta=(\log x)^{-f}$ with some $f$ in $b<f<1$, then there exists a positive constant $B$ such that

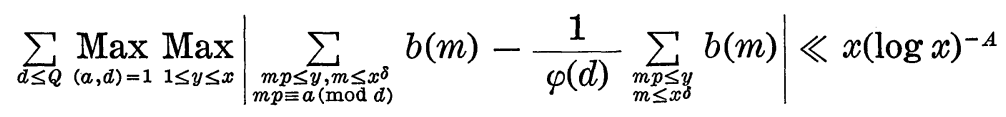

uniformly for $\delta$ in $0 \leq \delta<1-(\log x)^{-b}$, where $Q=x^{1 / 2}(\log x)^{-B}$. Using this, Theorems 3 and $3^{\prime}$ and Hooley's argument in [8], we can show an asymptotic formula for the number of the solutions of the equation

$$
N=p_{1} p_{2}+x^{2}+y^{2} \quad \text { for } p_{1} p_{2} \leq N .
$$

We do not need Linnik's dispersion method. (Cf. [11] and [12] for a proof of this using the dispersion method.) As is seen in [11] or [12] we may improve the remainder term in Theorem 2 if we use the dispersion method.

\section{REFERENCES}

[1] M. B. Baran, The large sieve method and its applications in the theory of numbers, Uspehi Mat. Nauk 21 (1966), no. 1 (127), 51-102=Russian Math. Surveys 21 (1966), no. 1, 49-103. 
[2] J. Chen, On the representation of a larger even integer as the sum of a prime and the product of at most two primes, Sc. Sinica 16 (1973), 157-176.

[ 3 ] P. D. T. A. Elliot and H. Halberstam, Some applications of Bombieri's theorem., Mathematika 13 (1966), 196-203.

[4] A. Fujii, Some remarks on Goldbach's problem, Acta Arith XXXII (1976), 27-35.

[5] A. Fujii, A local study of some additive problems in the theory of numbers, Proc. of Japan Acad. vol. 52, no. 3 (1976), 113-115.

[6] P. X. Gallagher, Bombieri's mean value theorem., Mathematika 15 (1968), 1-6.

[ 7$]$ H. Halberstam and H. E. Richert, Sieve methods, Academic Press, 1974, London, New York, San Francisco.

[8] C. Hooley, On the representation of a number as the sum of two squares and a prime, Acta Math. 97 (1957), 189-210.

[9] A. K. Karsiev and A. B. Sokolovskii, The generalized problem of Titchmarsh divisors, Mat. Zamet. t.3, no. 2, (1968), 187-194.

[10] A. K. Karsiev, The generalized problem of Titchmarsh divisors, Izv. Akad. Nauk UzSSSR Ser. Fiz.-Mat. Nauk 13 (1969), no. 1, 69-70.

[11] Yu. V. Linnik, The dispersion method in binary additive problems Leningrad, 1961, $208 \mathrm{pp}$.

[12] A. A. Poljanskii, An analogue of the Hardy-Littlewood equation, Soviet Math. Dokl., vol. 9 (1968), no. 3, 587-590.

[13] K. Prachar, Primzahlverteilung, 1957, Springer Verlag, Berlin, Gottingen, Heidelberg.

[14] G. Rodriquez, Sul problema dei divisori di Titchmarsh, Boll. Uni. Mat. Ital. (3) 20 (1965), 358-366.

[15] E. C. Titchmarsh, A divisor problem, Rend. Circ. Mat. Palermo, 54 (1930), 414429.

[16] R. C. Vaughan, On the number of solutions of the equation $p=a+n_{1} n_{2} \cdots n_{k}$ with $a<p \leq x$., J. London Math. Soc. (2), 6 (1972), 43-55.

Department of Mathematics

Rikkyo University 